\title{
Specificities of Ischemic Stroke Risk Factors in Arab-Speaking Countries
}

\author{
Halim Abboud ${ }^{a, c}$ Leila Sissani ${ }^{c, f} \quad$ Julien Labreuchec, ${ }^{c, h}$ Antonio Arauz ${ }^{i}$ \\ Marie-Germaine Bousser ${ }^{d, g} \quad$ Alain Bryer $^{j} \quad$ Angel Chamorro $^{k}$ Marc Fisher $^{1}$ \\ lan Ford ${ }^{m}$ Kim M. Fox ${ }^{n}$ Michael G. Hennerici ${ }^{p}$ Pablo M. Lavados ${ }^{q}$ \\ Ayrton Massaro $^{r}$ Heinrich P. Mattle ${ }^{s}$ Mario Munoz Collazos ${ }^{t}$ \\ Peter M. Rothwello Philippe Gabriel Steg ${ }^{f, g, n} \quad$ Eric Vicaut ${ }^{e, g}$ \\ Bassem Yamouth ${ }^{b}$ Pierre Amarenco ${ }^{c, f, g}$ on behalf of the OPTIC Registry
} and PERFORM Trial Investigators

\begin{abstract}
${ }^{a}$ Hotel Dieu de France, Saint Joseph University, and ${ }^{b}$ Department of Neurology, American University of Beirut Medical Center, Beirut, Lebanon; ' Department of Neurology and Stroke Centre, Hôpital Bichat, ${ }^{\mathrm{d}}$ Department of Neurology, Hôpital Lariboisière, and e Department of Biostatistics, Hôpital Fernand Widal, Assistance Publique-Hôpitaux de Paris, ${ }^{f}$ INSERM LVTS (Laboratory for Vascular Translational Sciences) 1148 and Département Hospitalo-Universitaire FIRE (Fibrosis, Inflammation, Remodelling), 9 Université Paris Diderot, Paris, and ${ }^{\text {h}}$ Université

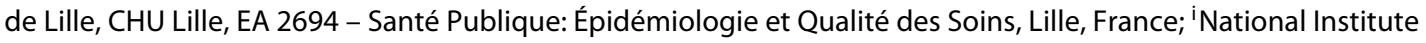
of Neurology, Mexico City, Mexico; jDivision of Neurology and Stroke Unit, Groote Schuur Hospital, University of Cape Town, Cape Town, South Africa; ${ }^{\mathrm{k}}$ Hospital Clinic de Barcelona, University of Barcelona, Barcelona,

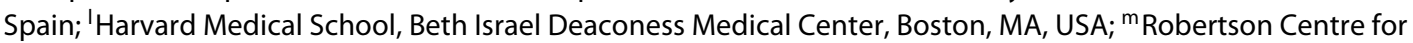
Biostatistics, University of Glasgow, Glasgow, ${ }^{\mathrm{N}} \mathrm{NHLI}$ Imperial College, ICMS, Royal Brompton Hospital, London,

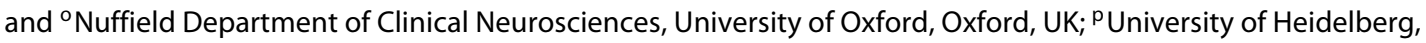
UMM, Mannheim, Germany; ${ }^{q}$ Unidad de Neurología Vascular, Servicio de Neurología, Departamento de Medicina, Clínica Alemana de Santiago, Facultad de Medicina, Clínica Alemana, Universidad del Desarrollo y Departamento de Ciencias Neurológicas, Facultad de Medicina, Universidad de Chile, Santiago, Chile; ${ }^{r}$ Neurologia, Hospital Sirio-Libanes, São Paolo, Brazil; ${ }^{5}$ Neurologische Klinik und Poliklinik, Universität Bern, Inselspital, Bern, Switzerland; ${ }^{\mathrm{t} N}$ Neurologia, Clinica de Marly, Bogota, DC, Colombia
\end{abstract}

\section{Keywords}

Stroke · TIA · Risk factors · Socio-economic status

\begin{abstract}
Background: Stroke is largely preventable, and therefore, a better understanding of risk factors is an essential step in reducing the population stroke rate and resulting disease burden in Arab countries. Summary: We performed 2 separate
\end{abstract}

analyses in 2 similar populations of patients with noncardioembolic ischemic stroke. This first involved 3,635 patients in the Outcomes in Patients with TIA and Cerebrovascular disease (OPTIC) registry (followed for 2 years), with baseline collection of the usual risk factors and 5 socioeconomic variables (unemployment status, residence in rural area, living in fully serviced accommodation, no health-insurance coverage, and low educational level). The second involved patients in the PERFORM trial ( $n=19,100$ followed up for 2

\section{KARGER}

(c) 2017 S. Karger AG, Basel

E-Mail karger@karger.com

www.karger.com/ced
Pierre Amarenco, MD

Department of Neurology and Stroke Centre Bichat University Hospital

46 rue Henri Huchard, FR-75018 Paris (France)

E-Mail pierre.amarenco@aphp.fr 
years), with baseline collection of the usual risk factors and 1 socioeconomic variable (low educational level). The primary outcome was a composite of nonfatal stroke, nonfatal myocardial infarction, or cardiovascular death. Stroke risk factors were more prevalent in patients in Arab countries. The incidence of major cardiovascular events (MACE; age- and gender-adjusted) was higher in Arab countries (OPTIC, 18.5 vs. 13.3\%; PERFORM, 18.4 vs. $9.7 \%$; both $p \leq 0.0001$ ). These results remained significant after adjustment on risk factors and were attenuated in OPTIC after further adjustment on socioeconomic variables (hazard ratio $1.24 ; 95 \% \mathrm{Cl} 0.98-$ $1.55 ; p=0.07)$. Key Messages: Patients with ischemic stroke living in Arab countries had a lower mean socioeconomic status, a much higher prevalence of diabetes mellitus, and a higher rate of MACE compared with patients from non-Arab countries. This finding is partly explained by a higher prevalence of risk factors and also by a high prevalence of poverty and low educational level.

๑ 2017 S. Karger AG, Basel

Atherothrombosis is a diffuse progressive process that manifests in multiple vascular beds; it starts early during childhood and progresses asymptomatically through adult life, resulting in nearly all cases of acute coronary syndrome and peripheral artery disease, and many cases of stroke [1]. Globally, stroke is the second leading cause of death [2] and over $80 \%$ of all stroke deaths worldwide occur in developing countries [3]. With epidemiology transition in low- and middle-income countries, more focus is projected on the control of atherosclerotic risk factors, but socioeconomic variables also play a major role in contributing to the risk of stroke [4].

Earlier on, the incidence of stroke was found to be the greatest in high-income countries, but its incidence in low- and middle-income countries now exceeds that of high-income countries by $20 \%[5,6]$. As stroke is largely preventable, knowledge of risk factors is an essential step in reducing the population stroke rate and resulting disease burden [7]. Many studies have addressed the specificities of risk factors in Asian countries, or across ethnicities, including black, Hispanic, and Asian ancestry, but no large studies have addressed the specificities of stroke risk factors and outcomes in Arab countries. We, therefore, performed parallel analyses of data from 2 international prospective studies that included similar populations of ischemic stroke patients, to compare risk factors (including socioeconomic factors) and 2-year outcomes between patients living in Arab (Middle East and North Africa) and non-Arab countries.

\section{Methods}

Data Source and Study Population

We first analyzed data from the Outcomes in Patients with TIA (transient ischemic attack) and Cerebrovascular disease (OPTIC) registry [4]. Briefly, the OPTIC registry enrolled 3,635 patients (January 2007-December 2008) age $\geq 45$ years from 17 low- and middle-income countries across Latin America (Brazil, Chile, Colombia, Dominican Republic, Ecuador, Mexico, Peru, Venezuela), the Middle East (Egypt, Iran, Jordan, Lebanon, Saudi Arabia), North Africa (Algeria, Morocco, Tunisia), and South Africa. Of these patients, 148 had no follow-up information, 130 did not return after the baseline visit, and 18 withdrew their consent to participate for other reasons, leaving a study population of 3,487 patients. OPTIC was designed to provide 2 -year clinical follow-up. Patients were followed every $6 \pm 1$ months for key outcome events, medication use, and change in employment status. The group from the Arab region included patients enrolled in countries in the Middle East and North Africa, except Iran.

We also analyzed data from patients enrolled in the Prevention of Cerebrovascular and Cardiovascular Events of Ischemic Origin with Terutroban in the Patients with a History of Ischemic Stroke or Transient Ischemic Attack (PERFORM) trial, an international randomized controlled trial, designed to compare terutroban with aspirin in the prevention of cerebral and cardiovascular ischemic events in patients ( $\geq 55$ years) with a noncardioembolic ischemic stroke ( $\leq 3$ months) or a TIA ( $\leq 8$ days) [8]. In short, PERFORM enrolled 19,100 subjects (February 2006-April 2008) from 802 sites in 46 countries over 5 continents. Follow-up visits were performed at 1, 3, and 6 months, and every 6 months thereafter; the minimum follow-up duration was 2 years. The group from the Arab region comprised patients enrolled in Morocco and Tunisia.

All participants provided written informed consent. We used the World Bank classification for the definition of Arab countries.

\section{Evaluations}

The baseline data collected for each patient included demographic characteristics, medical history, and medication use. In the OPTIC registry, socioeconomic profile (living alone, residence in rural area, living in fully serviced accommodation [i.e., house/ apartment with electricity, toilet, water supply], employment status, health insurance coverage, low education level [i.e., $<2$ years of schooling]) was also recorded. Education level was the only socioeconomic information collected in the PERFORM trial. Information, which was recorded at baseline and at follow-up along with key outcome events using a standardized case report form, was entered into a central database.

The primary study outcome was a composite of nonfatal stroke, nonfatal myocardial infarction, or cardiovascular death. Secondary outcomes were myocardial infarction and stroke separately (fatal or nonfatal). Events were not adjudicated in the OPTIC registry, whereas all events were adjudicated by a blinded endpoint committee in the PERFORM trial.

\section{Statistical Analysis}

Categorical variables are expressed as means \pm SD in the case of normal distribution or median (interquartile range [IQR]) otherwise. Qualitative variables are expressed as counts (percentage). Normality of distributions was assessed graphically and by using the Shapiro-Wilk test. All analyses were conducted separately in the 
OPTIC and PERFORM cohorts using the same analysis strategy. In both studies, patients were classified into 2 groups based on their country of origin (i.e., whether they resided in a Arab or a non-Arab country). Baseline characteristics (demographics, medical history, examination findings, socioeconomic factors, medication use) were compared between the 2 groups after adjustment on age and gender using linear regression models for quantitative variables and logistic regression models for binary variables. Owing to skewed distributions for triglycerides, creatinine, and glucose concentrations, linear regression analysis was performed using log-transformed values.

We compared the 2-year event rate (primary and secondary outcomes) between the 2 groups using Cox proportional hazard models adjusted for age and gender (model 1). Adjusted event curves and rates were calculated using the corrected-group prognosis method [9]; adjustment for age was made using the quartile values. For a given outcome, deaths that were not included in the endpoint were treated as censoring events. Events that occurred after the 2-year follow-up were not included. Using non-Arab countries as the reference, we derived, from Cox proportional hazard models, hazard ratios (HRs) as effect size measures with their 95\% CIs. The proportional hazard assumptions were checked using the log-log survival plots and by introducing a time-dependent variable into models.

Further multivariable Cox's regression models were performed to adjust the comparison in outcomes between the 2 study groups on traditional cardiovascular risk factors regarding the high disparities in their distribution between the study groups whatever the cohort. Similarly, regarding our previous OPTIC publication [10] showing an independent association of socioeconomics factors with cardiovascular risk, a multivariable model was performed by including socioeconomics factors as covariables. Thus, the following additional models were considered, which included model 1 variables plus hypertension, dyslipidemia, and smoking (model 2); model 2 variables plus body mass index and diabetes (model 3); and model 3 variables plus socioeconomic data (model 4). We also included these factors as a quantitative variable by calculating the total number, and evaluated its effect on the primary outcome across the 2 groups.

Finally, we combined the 2 datasets and performed the same analysis. All Cox's regression models were stratified on study.

Statistical testing was done, and a 2 -tailed $\alpha$-level of 0.05 was considered significant. Data were analyzed using SAS software version 9.3 (SAS Institute, Cary, NC, USA).

\section{Results}

Of the 3,487 patients in the OPTICregistry, 1,669(47.9\%) were from Arab countries and 1,818 from non-Arab countries; patients were followed for a median of 24 months (IQR 3-34 months). Of the 19,100 patients included in the PERFORM trial, 268 (1.4\%) were from Arab countries; the median follow-up was 29 months (IQR 24-34).

\section{Baseline Characteristics}

Table 1 shows the main baseline demographic and clinical history characteristics of patients in the OPTIC registry and the PERFORM trial. In both databases, the biggest difference was observed for diabetes. Patients from Arab countries had a higher prevalence of diabetes in both the OPTIC registry (43.9 vs. $29.3 \%, p<0.0001)$ and the PERFORM trial (50.0 vs. $27.4 \%, p<0.0001$ ). There were also significant differences in socioeconomic characteristics between Arab and non-Arab countries (Table 2). Online supplementary Table 1 (for all online suppl. material, see www.karger.com/doi/10.1159/000454776) shows high disparities in medication use at baseline across the 2 groups. The use of antiplatelet agents was more common at baseline in patients living in Arab countries. However, despite the high use of antiplatelet agents in primary and secondary prevention, and the good adherence to treatment during the study period, stroke continued to strike among the Arab region.

\section{Two-Year Vascular Event Rates}

In the OPTIC registry, 516 patients had at least 1 major cardiovascular event (MACE; nonfatal stroke, nonfatal myocardial infarction, or cardiovascular death) during the 2-year follow-up, with an event rate of $15.6 \%$ (95\% CI 14.4-16.9). The rate of MACEs for patients living in Arab countries was higher than in those living in non-Arab countries (age- and gender-adjusted HR 1.31; 95\% CI $1.08-1.58 ; p=0.006$; Fig. 1a). After adjustments on age, gender, hypertension, dyslipidemia, and current smoking (model 2), the results remained significant (HR 1.33; 95\% CI 1.09-1.63; $p=0.005$ ). When body mass index and diabetes were added to the model (model 3), the results remained unchanged (HR 1.32; 95\% CI 1.07-1.63; $p=$ $0.009)$. However, after adjusting for all variables plus socioeconomic variables, the association was no longer significant (HR 1.24; 95\% CI 0.98-1.55; $p=0.07$ ). When analyzing the outcome as a function of the number of socioeconomic factors, there were no changes in association between primary events and the 2 groups (adjusted HR 1.15 ; 95\% CI $0.93-1.43 ; p=0.20$ ). Patients with low socioeconomic factors were more frequently from Arab countries (Fig. 2a). In both Arab and non-Arab countries, the 2-year risk of MACEs increased with the number of low socioeconomic factors (age- and gender-adjusted $p$ value for trend $<0.0001$ for both; Fig. 2b). For Arab countries, the rate ranged from $15.8 \%$ for patients with no low socioeconomic factors to $56.9 \%$ in those with $\geq 5$ factors.

In the PERFORM trial, 1,833 patients had a MACE during the 2-year follow-up. As in the OPTIC registry, the 2 -year risk of vascular events was higher in patients from Arab countries (age- and gender-adjusted HR 1.97; 95\% CI 1.46-2.66; $p<0.0001$; Fig. 1b). The same results were obtained after multiple adjustments (Table 3 ). These re- 
Table 1. Baseline characteristics of patients living in Arab and non-Arab countries in the OPTIC registry and the PERFORM trial

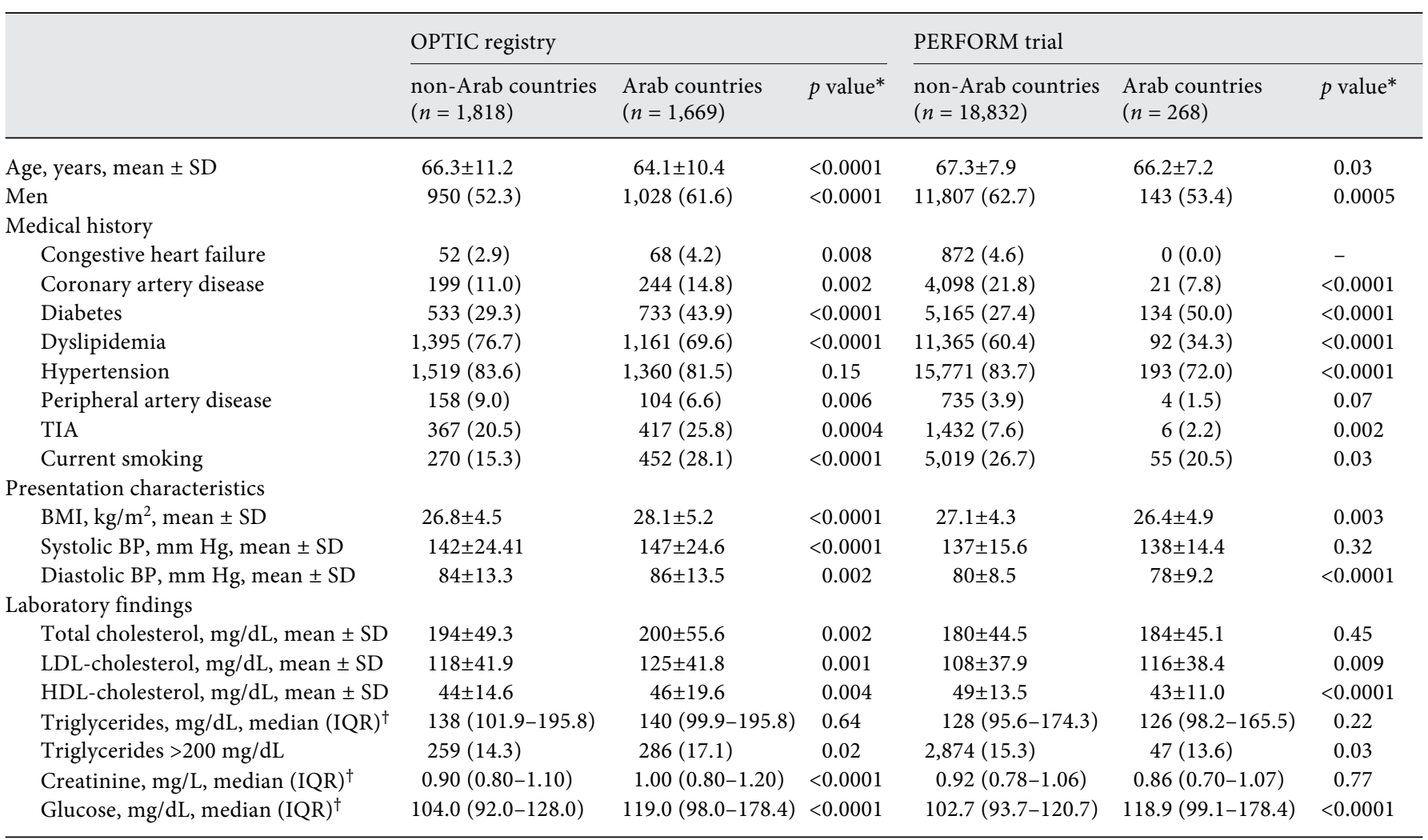

Data are number (\%) unless otherwise indicated.

TIA, transient ischemic attack; BMI, body mass index; BP, blood pressure; HDL, high-density lipoprotein; IQR, interquartile range; LDL, low-density lipoprotein. Percentage are based on available data.

* Age and gender-adjusted $p$ value.

${ }^{\dagger}$ Logarithmic values.

Table 2. Socioeconomic profile of patients living in Arab and non-Arab countries in the OPTIC registry and the PERFORM trial

\begin{tabular}{|c|c|c|c|c|c|c|}
\hline & $\begin{array}{l}\text { non-Arab countries } \\
(n=1,818)\end{array}$ & $\begin{array}{l}\text { Arab countries } \\
(n=1,669)\end{array}$ & $p$ value* & $\begin{array}{l}\text { non-Arab countries } \\
(n=18,832)\end{array}$ & $\begin{array}{l}\text { Arab countries } \\
(n=268)\end{array}$ & $p$ value* \\
\hline Living alone & $194(10.8)$ & $98(5.9)$ & $<0.0001$ & NA & NA & \\
\hline Living in rural area & $130(7.2)$ & $310(19.0)$ & $<0.0001$ & NA & NA & \\
\hline Unemployed $^{\dagger}$ & $484(26.6)$ & $600(36.0)$ & $<0.0001$ & NA & NA & \\
\hline No health insurance coverage & $266(14.7)$ & $533(32.0)$ & $<0.0001$ & NA & NA & \\
\hline Low educational level ${ }^{\ddagger}$ & $210(11.6)$ & $692(42.5)$ & $<0.0001$ & $2,611(13.9)$ & $232(67.0)$ & $<0.0001$ \\
\hline
\end{tabular}

Data are number (\%).

* Age- and gender-adjusted $p$ value.

† Excluding social pensioner on disability grant or old-age pensioner.

₹ The threshold was $<2$ years of schooling in OPTIC and $\leq 4$ years of schooling in PERFORM.

NA indicates not available. 


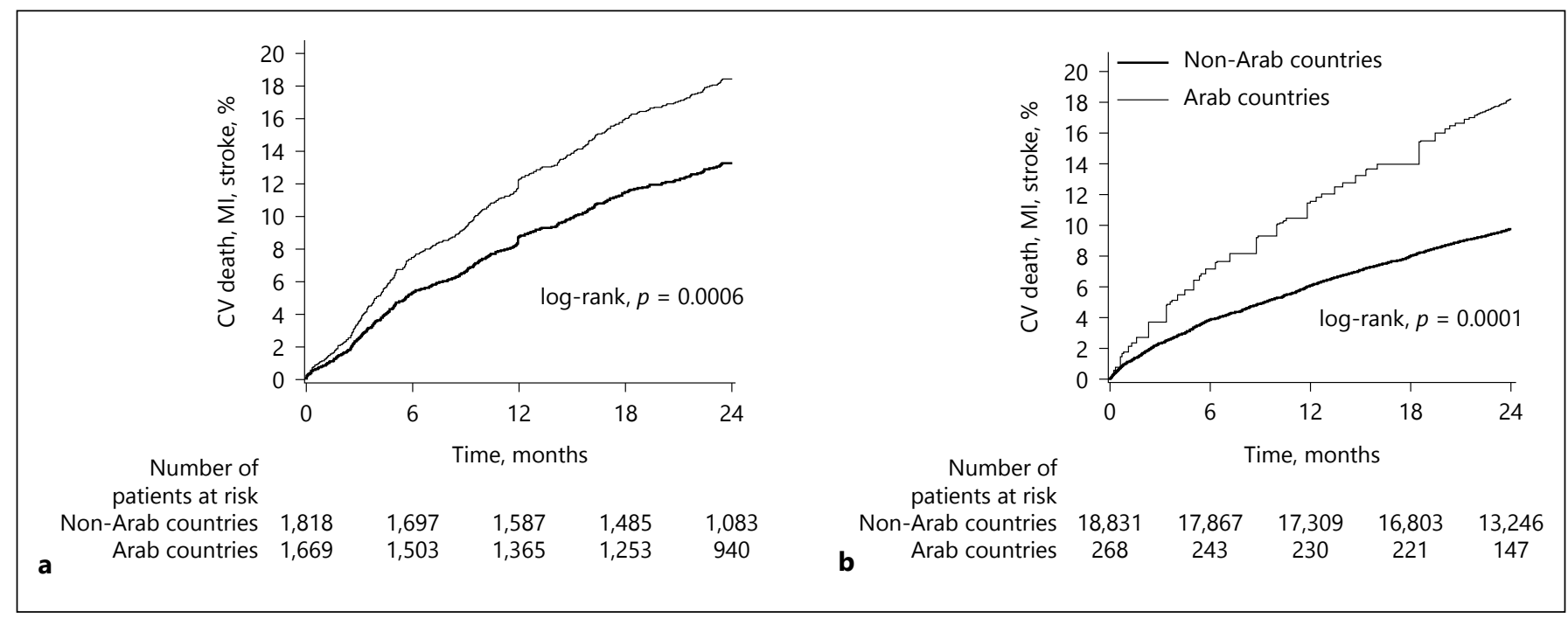

Fig. 1. Cumulative incidence curves for nonfatal stroke, nonfatal myocardial infarction, or cardiovascular death in Arab and nonArab countries to the 2 groups in the OPTIC registry (a) and the

sults are partly explained by a large difference in the rate of vascular deaths between the 2 groups, which was 5 times higher in patients living in Arab countries. This finding should, however, be interpreted with caution due to the low proportion of patients living in Arab countries enrolled in the PERFORM trial (1.4\%).

In the combined analysis, we found a significant association between the 2 groups regarding the risk of MACE, fatal, or nonfatal stroke and cardiovascular death in all the models tested (Table 3). The risk was higher among patients living in Arab countries as against those not living in Arab countries.

\section{Discussion}

Thelarge, multinational OPTIC registry and PERFORM trial provided an opportunity to evaluate stroke risk factors in patients from Arab countries. We found a trend for a higher frequency of the usual risk factors for stroke and evidence of inadequately managed diabetes mellitus, hypertension, and tobacco smoking in patients from Arab countries. Furthermore, stroke appeared to occur at a younger age in patients living in Arab countries, as reported previously [11-13], indicating an opportunity to prevent the occurrence of premature stroke. Another important finding from our analysis was that after adjustment on socioeconomic variables, the differences between patients living in Arab and non-Arab countries were attenuated in the OPTIC
PERFORM trial (b). Adjusted event curves were calculated using the corrected group prognosis after categorization of age into quartile.

registry, indicating that these socioeconomic variables significantly affect stroke risk in these countries. Our findings suggest that improvements in healthcare systems and educational level, and provision of fully serviced accommodation and health insurance cover in Arab countries may help to reduce the incidence of stroke.

In our study, the prevalence of hypertension was higher in non-Arab countries than in Arab countries. One probable reason for this is the older mean age of patients with stroke in non-Arab countries, since blood pressure tends to increase with age [12]. Additionally, hypertension tends to occur more frequently in Arab women compared with Arab men [13]. Finally, limited access to healthcare services and lack of preventive care may contribute to low hypertension awareness and high hypertension at the time of admission and antihypertensive medications at follow-up in patients living in Arab countries (online suppl. Table 2).

Diabetes, as an independent risk factor for stroke, was highly prevalent in Arab countries. The prevalence was $43.9 \%$ in the OPTIC registry and $50.0 \%$ in PERFORM trial, as compared with 29.3 and $27.4 \%$, respectively, in non-Arab countries. Diabetes is also reported to be more frequent in patients living in urban areas [14]. An estimated $9.1 \%$ of the population from the Middle Eastern/North African region was estimated to have type 2 diabetes (32.8 million people) in 2011, and this epidemic is projected to reach 60 million in 2030. Many factors, from changes in physical activity and nutrition 
Fig. 2. Frequency of low socioeconomic factors among Arab and non-Arab countries in the OPTIC registry (a); age- and gender-adjusted event rates for nonfatal stroke, nonfatal myocardial infarction, or cardiovascular death, according to the number of low socioeconomic factors, in patients living in Arab vs. non-Arab countries in the OPTIC registry (b).

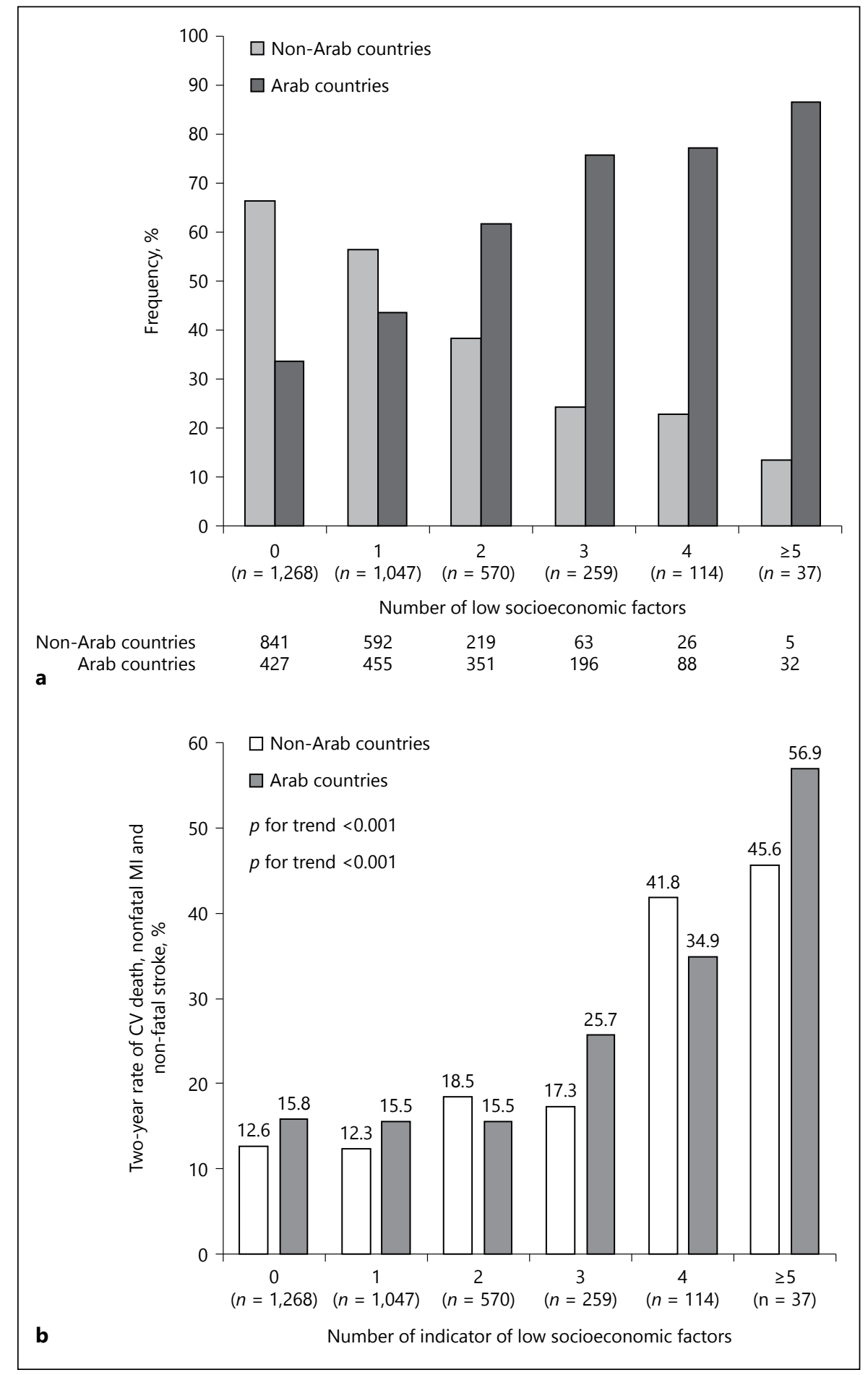

patterns [15] to genetic predisposition [16], are likely to contribute to the high prevalence of diabetes and overweight/obesity in this region. Consequently, one expects a high correlation between stroke in Arab regions and diabetes.
While rates of smoking have increased in most Arab countries, they have decreased in most western countries [17]. The overall percentage of cigarette smoking (current) was higher for stroke patients in the Arab region compared to non-Arab countries in the OPTIC registry 





( 28.1 vs. $15.3 \%$, respectively). In addition, tobacco use in many Arab countries is not limited to cigarettes. Waterpipe smoking is growing in popularity among the youth in the Middle East and North Africa, and even worldwide [18]. Another issue is smoking in women, which is often construed as a vice that undermines the social standing of the family in many Muslim communities [19]. As a result, the prevalence of smoking in the Arab region is probably underestimated in our study.

Antiplatelet agents were used in 95\% of [0] patients living in Arab countries as compared to $91 \%$ in other OPTIC registry countries. However, despite the high use of antiplatelet agents in primary and secondary prevention, and the good adherence to treatment during the study period, occurrence of stroke continue to strike among Arab region.

In the OPTIC registry, stroke survivors in Arab countries lived more frequently in rural areas with their families, they were also less likely to have health insurance cover or to achieve a high educational level than patients in non-Arab countries. This presents another barrier to stroke prevention because higher educational level correlates with increased awareness of stroke vascular risk factors [20], and particularly about lifestyle choices. This study was limited by the fact that we were unable to analyze the patients according to their ethnicity. Second, this study may not be entirely representative of the Arab countries, which exhibit variations in educational level, economic factors, and health improvements.
In conclusion, patients with ischemic stroke living in Arab countries had a lower mean socioeconomic status, a much higher prevalence of diabetes mellitus, and a higher risk of recurrent vascular events compared with patients from non-Arab countries. These differences were markedly attenuated after adjustment on socioeconomic variables. Thus, there is an opportunity for major improvements in the prevention of stroke and cardiovascular events in these countries, at the health policy level as well as with primary prevention measures, including health policy for smoking cessation and lifestyle changes to modify the metabolic profile of the population and to lower blood pressure. There is, however, an urgent need for comprehensive studies on the role of low socioeconomic status and its contribution to the occurrence of stroke and cardiovascular events in Arab countries.

\section{Acknowledgments}

Editorial support was provided by Sophie Rushton-Smith, $\mathrm{PhD}$, and was funded by SOS-Attaque Cerebrale Association.

\section{Sources of Funding}

This was an investigator-driven initiative and was funded by SOS-Attaque Cerebrale Association (a not-for-profit Stroke survivors Association). The OPTIC study was funded by Sanofi and the PERFORM trial was funded by Servier.

\section{References}

1 Leys D: Atherothrombosis: a major health burden. Cerebrovasc Dis 2001;11(suppl 2):14

2 Murray CJ, Lopez AD: Mortality by cause for eight regions of the world: Global Burden of Disease Study. Lancet 1997;349: 1269-1276.

3 Murray CJ, Lopez AD, Chin B, Feehan D, Hill $\mathrm{KH}$ : Estimation of potential global pandemic influenza mortality on the basis of vital registry data from the 1918-20 pandemic: a quantitative analysis. Lancet 2006;368:2211-2218.

4 Abboud H, Labreuche J, Arauz A, Bryer A, Lavados PG, Massaro A, et al: Demographics, socio-economic characteristics, and risk factor prevalence in patients with non-cardioembolic ischaemic stroke in low- and middleincome countries: the OPTIC registry. Int J Stroke 2013;8(suppl A100):4-13.

5 Lackland DT, Roccella EJ, Deutsch AF, Fornage $\mathrm{M}$, George MG, Howard G, et al: Factors influencing the decline in stroke mortality: a statement from the American Heart Association/American Stroke Association. Stroke 2014;45:315-353.

6 Feigin VL, Lawes CM, Bennett DA, BarkerCollo SL, Parag V: Worldwide stroke incidence and early case fatality reported in 56 population-based studies: a systematic review. Lancet Neurol 2009;8:355-369.

7 EUROASPIRE II Study Group: Lifestyle and risk factor management and use of drug therapies in coronary patients from 15 countries; principal results from EUROASPIRE II Euro Heart Survey Programme. Eur Heart J 2001; 22:554-572.

8 Bousser MG, Amarenco P, Chamorro A, Fisher M, Ford I, Fox K, et al: The prevention of cerebrovascular and cardiovascular events of ischemic origin with terutroban in patients with a history of ischemic stroke or transient ischemic attack (PERFORM) study: baseline characteristics of the population. Cerebrovasc Dis 2009;27:608-613.
9 Ghali WA, Quan H, Brant R, van Melle G, Norris CM, Faris PD, et al: Comparison of 2 methods for calculating adjusted survival curves from proportional hazards models. JAMA 2001;286:1494-1497.

10 Amarenco P, Abboud H, Labreuche J, Arauz A, Bryer A, Lavados PM, et al: Impact of living and socioeconomic characteristics on cardiovascular risk in ischemic stroke patients. Int J Stroke 2014;9:1065-1072.

11 Tran J, Mirzaei M, Anderson L, Leeder SR: The epidemiology of stroke in the middle East and North Africa. J Neurol Sci 2010;295:3840.

12 Wolf-Maier K, Cooper RS, Banegas JR, Giampaoli S, Hense HW, Joffres M, et al: Hypertension prevalence and blood pressure levels in 6 European countries, Canada, and the United States. JAMA 2003;289:2363-2369.

13 Addo J, Smeeth L, Leon DA: Hypertension in sub-saharan Africa: a systematic review. Hypertension 2007;50:1012-1018. 
14 Badran M, Laher I: Type II diabetes mellitus in Arabic-speaking countries. Int J Endocrinol 2012;2012:902873.

15 Rahim HF, Sibai A, Khader Y, Hwalla N, Fadhil I, Alsiyabi H, et al: Non-communicable diseases in the Arab world. Lancet 2014;383:356-367.

16 Meyer BF, Alsmadi O, Wakil S, Al-Rubeaanb $\mathrm{K}$ : Genetics of type 2 diabetes in Arabs: what we know to date. Int J Diabetes Mellitus 2009; 1:32-34.
$17 \mathrm{Ng}$ M, Freeman MK, Fleming TD, Robinson M, Dwyer-Lindgren L, Thomson B, et al: Smoking prevalence and cigarette consumption in 187 countries, 1980-2012. JAMA 2014;311:183-192.

18 Maziak W, Ward KD, Afifi Soweid RA, Eissenberg T: Tobacco smoking using a waterpipe: a re-emerging strain in a global epidemic. Tob Control 2004;13:327-333.
19 Bush J, White M, Kai J, Rankin J, Bhopal R Understanding influences on smoking in Bangladeshi and Pakistani adults: community based, qualitative study. BMJ 2003;326: 962

20 Muller-Nordhorn J, Nolte CH, Rossnagel K: Knowledge about risk factors for stroke: a population-based survey of 28,090 participants. Stroke 2006;37:946-950. 